\title{
National Institute on Drug Abuse
}

National Cancer Institute

\section{Source}

National Cancer Institute. National Institute on Drug Abuse. NCI Thesaurus. Code C19757.

An institute within the National Institutes of Health with the mission to lead the nation in bringing the power of science to bear on drug abuse and addiction. This charge has two critical components, the strategic support and conduct of research across a broad range of disciplines and the rapid and effective dissemination and use of the results of that research to significantly improve prevention, treatment and policy as it relates to drug abuse and addiction. 